Pacific Journal of Mathematics

SCHWARZ NORMS FOR OPERATORS 


\title{
SCHWARZ NORMS FOR OPERATORS
}

\author{
JAMES P. WILLIAMS
}

Let $H$ be a complex Hilbert space and let $\mathscr{L}(H)$ be the algebra of bounded linear operators from $H$ into itself. A norm | | on $\mathscr{L}(H)$ which is equivalent to the usual norm will be called a Schwarz norm if the following version of the Schwarz lemma is valid for | |:

Schwarz Lemma If $f$ is analytic and bounded by 1 in $|z|<1$, and if $f(0)=0$, then $|f(T) \leqq| T \mid$ for each operator $T$ with $|T|<1$.

It is an immediate consequence of the classical lemma that equality holds for some $T$ if and only if $f(z) \equiv \gamma z$ for some constant $\gamma$.

Von Neumann [5] proved that the usual norm, which we will call ||$_{2}$, is a Schwarz norm, and recently, Berger and Stampfli [1] proved that the numerical radius norm

$$
|T|_{1}=|W(T)|=\sup \{|<T x, x>|:\|x\|=1\}
$$

is also a Schwarz norm. It is our purpose here to point out that a slight modification of the Berger-Stampfli argument produces a family | $\left.\right|_{c}(c \geqq 1)$ of distinct Schwarz norms on $\mathscr{L}(H)$. We will also mention some negative results concerning Schwarz norms in $\mathscr{L}(X)$ when $X$ is a Banach space.

1. It is a simple matter to check that the preceding version of the Schwarz lemma may be equivalently stated in the following form:

SchWARZ LEMma If $|T| \leqq 1$, then $|f(T)| \leqq\|f\|$ for each $f \in R(D)$ such that $f(0)=0$.

Here $R(D)$ is the (sup-norm) algebra of rational functions with no poles in the closed unit disk $D$ and $f(T)$ is defined by the usual Cauchy integral around a circle slightly larger than the unit circle.

An elementary computation shows that for $|\alpha|<1$ the conformal map $\varphi_{\alpha}(z)=(z-\alpha)(1-\bar{\alpha} z)^{-1}$ takes contractions into contractions [5]. This implies that the second version of the Schwarz lemma for ||$_{2}$ is actually valid without the condition $f(0)=0$. On the other hand, this condition cannot be omitted for the numerical radius norm as the following result shows:

THeorem 1. $\left|\varphi_{\alpha}(T)\right|_{1} \leqq 1$ for all $|\alpha|<1$ if and only if $|T|_{2} \leqq 1$. 
Proof. Since ||$_{1} \leqq||_{2}$, one half of the implication is clear. To get the other half, note that the hypothesis implies that

$$
|<(T-\alpha) x,(1-\bar{\alpha} T) x>| \leqq\|(1-\bar{\alpha} T) x\|^{2}
$$

for all $x \in H$ and all $|\alpha|<1$. Expanding this one concludes that

$$
\begin{aligned}
\lambda\left(\|x\|^{2}+\|T x\|^{2}\right) & +\left(\lambda^{2}+1\right) \mid<T x, x>1 \\
& \leqq\|x\|^{2}+2 \lambda|<T x, x>|+\lambda^{2}\|T x\|^{2}
\end{aligned}
$$

for $x \in H$ and $0 \leqq \lambda<1$. Hence

$$
0 \leqq(\lambda-1)\left[\lambda\left(\|T x\|^{2}-|<T x, x>|\right)-\left(\|x\|^{2}-|<T x, x>|\right)\right]
$$

and since the expression in the bracket is therefore nonpositive for $0<\lambda<1$, it follows that $|T|_{2}=\|T\| \leqq 1$.

2. It is well-known that if the spectrum of $T$ lies in $D$, then $\operatorname{Re}(1+z T)(1-z T)^{-1} \geqq 0$ all $|z|<1$ and $\operatorname{Re}(1-z T)^{-1} \geqq 0$ all $|z|<1$ are respectively equivalent to $\|T\| \leqq 1,|W(T)| \leqq 1$. Here the relevent analytic functions have series expansions of the form $1+c \sum_{1}^{\infty} z^{n} T^{n}$ (with $c=2$ and $c=1$ ) and so we are led to introduce the following class of operators:

Definition. For $c>0$ let $S_{c}$ be the class of operators $T$ with $\sigma(T) \subset D^{1}$ for which $\operatorname{Re}\left(1+c \sum_{1}^{\infty} z^{n} T^{n}\right) \geqq 0$ in $|z|<1$. Thus $T \in S_{2}$ $\left(T \in S_{1}\right)$ if and only if $|T|_{2}=|| T|| \leqq 1\left(|T|_{1}=|W(T)| \leqq 1\right.$ ) so that the following theorem includes the results of Von Neumann and BergerStampfli:

Theorem 2. If $T \in S_{c}(c>0)$ then $f(T) \in S_{c}$ for each $f \in R(D)$ satisfying $f(0)=0$ and $\|f\|_{D} \leqq 1$.

Proof. The proof is essentially the Berger-Stampfli argument: (For a slightly different proof see [4].) If $T \in S_{c}$, then by a theorem of Herglotz, there is a positive measure $\mu_{x}$ on $[0,2 \pi]$ such that

$$
\|x\|^{2}+c \sum_{1}^{\infty} z^{n}<T^{n} x, x>=\int \frac{1+z e^{i \theta}}{1-z e^{i \theta}} d \mu_{x}(\theta)
$$

for all $|z|<1$. Expanding the integrand and equating coefficients we get

$$
c<T^{n} x, x>=2 \int e^{i n \theta} d \mu_{x}(\theta) \quad(n \geqq 1) .
$$

1 This condition insures that the series converges in $|z|<1$. 
If now $f(z)=\sum_{1}^{n} \alpha_{k} z^{k}$ is a polynomial, then Eq. $(*)$ implies

$$
c<f(T) x, x>=2 \int f\left(e^{i \theta}\right) d \mu_{x}(\theta) .
$$

Applying this fact to $f^{n}$ we obtain

$$
c<f(T)^{n} x, x>=2 \int f^{n}\left(e^{i \theta}\right) d \mu_{x}(\theta) \quad(n \geqq 1) .
$$

It follows that if $\|f\| \leqq 1$, then $\left\|f(T)^{n}\right\|$ is bounded so that for $|z|<1$,

$$
\begin{aligned}
<\left(1+c \sum z^{n} f(T)^{n}\right) x, x> & =\|x\|^{2}+2 \sum_{1}^{\infty} z^{n} \int f\left(e^{i \theta}\right)^{n} d \mu_{x}(\theta) \\
& =\int \frac{1+z f\left(e^{i \theta}\right)}{1-z f\left(e^{i \theta}\right)} d \mu_{x}(\theta) .
\end{aligned}
$$

The integrand has positive real part in $|z|<1$ and therefore $f(T) \in S_{c}$. To complete the proof, note that each $f \in R(D)$ can be uniformly approximated by polynomials on a disk of radius $1+\varepsilon$.

REMARK. It is easy to proceed from Eq. $(*)$ via polarization and Naimark's theorem on the dilation of an operator measure to obtain a "2/c-unitary dilation" of $T \in S_{c}$, i.e., to obtain a unitary operator $U$ on a larger Hilbert space $K$ such that

$$
T^{n}=2 / c P U^{n} \mid H \quad(n \geqq 1)
$$

where $P$ is the orthogonal projection of $K$ onto $H$. This is the approach of Nagy-Foias in [4]. The class $S_{c}$ is their class $\mathscr{C}_{2 / c}$.

3. The next proposition gives some information about the class $S_{c}$.

Theorem 3. Let $c>0$. Then

(i) $S_{c}^{*}=S_{c}$.

(ii) $\varphi \neq S_{c^{\prime}} \varsubsetneqq S_{c}$ if $c<c^{\prime}$.

(iii) $S_{c}$ is convex if and only if $c \geqq 1$.

(iv) $T \in S_{c}(c \geqq 1)$ if and only if

$$
(c-1)\|T x\|^{2}+|2-c| \mid<T x, x>\leqq\|x\|^{2}
$$

all $x \in H$.

Proof. By deffinition, we have $T \in S_{c}$ if and only if $\sigma(T) \subset D$ and $\operatorname{Re}<(1-z T)^{-1} x, x>\geqq\left(1-c^{-1}\right)\|x\|^{2}$ for all $x \in H$ and all $|z|<1$. The first assertion and the inclusion in (ii) follow immediately from this. Next, observe that if $c \geqq 1$, then the condition $T \in S_{c}$ is equivalently 
written

$$
\operatorname{Re}<y,(1-z T) y>\geqq \frac{c-1}{c}\|(1-z T) y\|^{2}
$$

for all $y \in H$ and all $|z|<1$, and (iv) now follows by a simple calculation.

Using (iv), it is easy to see that, for $c \geqq 1, S_{c}$ is weakly closed, bounded, circled and a (norm) neighborhood of 0 . Also by the parallelogram law,

$$
\left\|\frac{1}{2} T_{1} y+\frac{1}{2} T_{2} y\right\|^{2} \leqq \frac{1}{2}\left(\left\|T_{1} y\right\|^{2}+\left\|T_{2} y\right\|^{2}\right)
$$

and this implies that $\frac{1}{2} S_{c}+\frac{1}{2} S_{c} \subset S_{c}$. Since any closed midpoint-convex set is convex, it follows that $S_{c}$ is convex for $c \geqq 1$.

It remains to see that the classes $S_{c}$ are nonempty, strictly decreasing, and if $c<1$, not convex. To prove these facts, let $A$ be the two-dimensional operator with matrix $\left(\begin{array}{l}00 \\ 10\end{array}\right)$. If $s>0$, then $s A$ has spectrum $\{0\}$ and is nilpotent of index 2 ; hence if $c>0$ then $s A_{2} \in S_{c}$ if and only if

$$
0 \leqq \operatorname{Re}\left(1+c \sum_{1}^{\infty} z^{n} s^{n} A^{n}\right)=\operatorname{Re}(1+c z s A) .
$$

For all $|z|<1$. Since the spectrum of $\operatorname{Re}(1+c z s A)$ consists of the points $1 \pm \frac{1}{2}|c z s|$, we conclude that $s A \in S_{c}$ if and only if $c s \leqq 2$.

It follows that if $c>0$, then $(2 / c) A \in S_{c}$. Also, if $c^{\prime}>c$, then

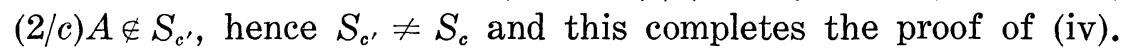
Finally, if $c>0$ and $S_{c}$ is convex, then $S_{c}$ must contain

$$
\frac{1}{2}\left(\frac{2}{c} A+\frac{2}{c} A^{*}\right)=\frac{2}{c} \operatorname{Re} A \text {. }
$$

But $(2 / c) \operatorname{Re} A$ has spectrum $\{-1 / c, 1 / c\}$ and therefore $1 / c \leqq 1$, which completes the proof of (iii).

From the properties of $S_{c}$ just mentioned it follows that for $c \geqq 1$, the gauge of $S_{c}$, i.e., the functional

$$
|T|_{c}=\inf \left\{\lambda>0: T \in \lambda S_{c}\right\},
$$

is a norm equivalent to the usual norm \|\|$=,\left.\right|_{2}$. Moreover Theorem 2 shows that each of these norms is a Schwarz norm.

THEOREM 4. Let $c \geqq 1$.

(i) $\left|T^{*}\right|_{0}=|T|_{0}$ for all $T$.

(ii) ||$_{c} \leqq\left. 1 \quad\right|_{c^{\prime}}$ if $c \leqq c^{\prime}$.

(iii) If $c<2$, then $|1|_{c}=1$, but ||$_{c}$ is not an algebra norm in $\mathscr{L}(H)$.

2 The same example is used in [4] to prove (ii). We include it here because of its use in (iii). 
(iv) $2|\quad|_{1} \leqq 2|\quad|_{c} \leqq(c+\mid 2-c)|\quad|_{2}$ and for $1 \leqq c \leqq 2$ these bounds are sharp.

(v) If $\left|\varphi_{\alpha}(T)\right|_{c} \leqq 1$ for all $|\alpha|<1$, then $|T|_{2} \leqq 1$.

(vi) If $|T|_{c} \leqq 1$ and $\left|T^{-1}\right|_{c} \leqq 1$, then $T$ is unitary.

Proof. The first two assertions are clear from Theorem 3. A computation shows that if $c \geqq 1$, then $|1|_{c}=(c+|2-c|) / 2$ (and hence $1 \in S_{c}$ if and only if $1 \leqq c \leqq 2$ ). To prove (iii) we appeal to the minimality of the norm in a $B^{*}$ algebra to see that if $|\quad|_{c} \leqq\left.\right|_{2}$ and if $\left\{\mathscr{L}(H),|\quad|_{c}\right\}$ is a normed algebra then ||$_{c}=|\quad|_{2}$, hence $c=2$.

To get the bound on the quotient $\left.||_{2}||\right|_{c}$, note that if $|T|_{c}=1$, then $(1+1 / n) T \notin S_{c}$, and hence by Theorem 3 there exists a sequence of unit vectors $x_{n}$ such that

$$
\begin{gathered}
(c-1)(1+1 / n)^{2}\left\|T x_{n}\right\|^{2}+|2-c|(1+1 / n)\left\|T x_{n}\right\| \\
\geqq(c-1)(1+1 / n)\left\|T x_{n}\right\|^{2}+|2-c|(1+1 / n)\left|<T x_{n}, x_{n}>\right|>1 .
\end{gathered}
$$

This implies that $|T|_{2}=\|T\| \geqq 2 /(c+|2-c|)$.

Assertions (v) and (vi) clearly follow from the case $c=1$, and if $c=1$ then (v) is true by Theorem 1 and (vi) is a theorem of Stampfli [6].

REMARKs. (1) For each $c \geqq 1$ the unit ball of the norm ||$_{c}$ is weakly compact and this implies that ||$_{c}$ is the dual of an equivalent norm on the pre-dual of $\mathscr{C}(H)$. However, ||$_{c}$ is either not multiplicative (for $c<2$ ) or does not assign the value 1 to the identity operator (for $c>2$ ) and hence none of these norms is induced by an equivalent norm on $H$.

(2) It seems quite likely that the norms ||$_{c}$ do not include all Schwarz norms on $\mathscr{L}(H)$, but I am unable to find another one.

4. In this concluding paragraph we will comment on the validity of the preceding results when the underlying space $H$ is replaced by a Banach space.

To being with, recall [3] that one may introduce a semi-innerproduct on any Banach space $X$ by choosing a map $\eta: X \rightarrow X^{*}$ satisfying

$$
<x, \eta(x)>=\|x\|^{2},\|\eta(x)\|=\|x\| \quad(x \in X) .
$$

and then defining

$$
[x, y]=\langle x, \eta(y)\rangle \quad(x, y \in X) .
$$

The definition of $S_{c}$ is meaningful relative to this semi-innerproduct and it is easy to check that Theorem 2 remains valid in this general setting. Indeed, the proof uses only linearity of the inner product with respect to its first argument, and the fact that the 
norm of an operator is dominated by a multiple of its numerical radius; this latter fact is shown in [3].

It is reasonable to ask whether the natural norm or the numerical radius norm is a Schwarz norm for $\mathscr{L}(X)$. Before answering these questions (negatively), the following remarks are offered as affirmative evidence:

(1) For the usual norm ||$_{2}$ it is known that the condition $f(0)=0$ cannot be omitted. Thus if each $\varphi_{\alpha}$ carries contractions to contractions, then the norm in $X$ satisfies the parallelogram law [2]. So the question really is concerned with the Schwarz Lemma.

(2) Theorem 2 gives an affirmative for the norm ||$_{1}$ provided that $\operatorname{Re} A \geqq 0$ implies $\operatorname{Re} A^{-1} \geqq 0$ for invertible operators $A \in \mathscr{L}(X)$. This seems plausible at first sight, but Example 1 below shows that the implication fails even when $X$ is a smooth Banach space (i.e., when $X$ has a unique semi-inner-product.)

ExAmple 1. Let $X$ be the two-dimentional complex Banach space with norm $\left\|\left(x_{1}, x_{2}\right)\right\|=\left(\left|x_{1}\right|^{P}+\left|x_{2}\right|^{p}\right)^{1 / p}$ where $p>1$. Then there are invertible operators $T$ on $X$ such that $\operatorname{Re} T \geqq 0$ and $\operatorname{Re} T^{-1} \geqq 0$.

Note first that $X$ is smooth and the mapping $\eta$ from $X$ into its dual is given by

$$
\eta\left(x_{1}, x_{2}\right)=\left(\bar{x}_{1}\left|x_{1}\right|^{p-2}, \bar{x}_{2}\left|x_{2}\right|^{p-2}\right)
$$

when $\left\|\left(x_{1}, x_{2}\right)\right\|=1$. The condition $\operatorname{Re} T \geqq 0$ means of course that $\operatorname{Re}<T x, \eta(x)>\geqq 0$ for all $x \in X$.

Proposition. Let $T=\left(\begin{array}{l}a 0 \\ c b\end{array}\right)$. Then $\operatorname{Re} T \geqq 0$ if and only if

(i) $\operatorname{Re} a \geqq 0, \operatorname{Re} b \geqq 0$

(ii) $|c| \leqq(p \operatorname{Re} a)^{1 / p}(q \operatorname{Re} b)^{1 / q}$

where $(1 / p)+(1 / q)=1$.

It follows that if $\operatorname{Re} T \geqq 0$, then $\operatorname{Re} T^{-1} \geqq 0$ if and only if

$$
|c / a b| \geqq(p \operatorname{Re} 1 / a)^{1 / p}(q \operatorname{Re} 1 / b)^{1 / q}
$$

or, if and only if

$$
|c| \leqq|a|^{1-2 / p}|b|^{1-2 / q}(p \operatorname{Re} a)^{1 / p}(q \operatorname{Re} b)^{1 / q} .
$$

It is therefore clear that $\operatorname{Re} T \geqq 0$ and $\operatorname{Re} T^{-1} \geqq 0$ are not equivalent. The proof of the Proposition is straightforward but somewhat complicated and will therefore be omitted. The case $p=2$ and the limiting case $p=1(|c| \leqq \operatorname{Re} a)$ however are easy to check.

ExAmple 2. Let $X$ be the two-dimensional Banach space with norm $\left\|\left(x_{1}, x_{2}\right)\right\|=\left|x_{1}\right|+\left|x_{2}\right|$. The natural norm in $\mathscr{L}(X)$ is not a 
a Schwarz norm.

The norm of the operator $\left(\begin{array}{l}a 0 \\ c b\end{array}\right)$ on $X$ is easily found to be

$$
\max \{|a|+|c|,|b|\} \text {. }
$$

Consequently, if $0<a<1$, then $T=\left(\begin{array}{cc}a & 0 \\ 1-a & 1\end{array}\right)$ is a contraction on $X$. To prove the assertion it will suffice to show that there is a complex number $\alpha$ of modulus less than 1 such that $\left\|\psi_{\alpha}(T)\right\|>1$ where $\psi_{\alpha}(z)=z(z+\alpha)(1+\bar{\alpha} z)^{-1}$.

The condition $\left\|\psi_{\alpha}(T)\right\| \leqq 1$ is equivalent to

$$
a\left|\frac{\alpha+a}{1+\bar{\alpha} a}\right|+(1-a)\left|\frac{1+\alpha+a(1+\bar{\alpha})}{(1+\bar{\alpha} a)(1+\alpha)}\right| \leqq 1
$$

which, for real $\alpha$ simplifies to

$$
a|\alpha+a|+(1-a)(1+a) \leqq|1+\alpha a| \text {. }
$$

This fails for $\alpha=-1 / 2(\alpha+1)$.

ExAmple 3. Let $X$ be the Banach space of Example 2, and define $\eta: X \rightarrow X^{*}$ on unit vectors as follows:

$$
\eta\left(x_{1}, x_{2}\right)= \begin{cases}\left(\operatorname{sgn} \bar{x}_{1}, \operatorname{sgn} \bar{x}_{2}\right) & \text { if } x_{1} x_{2} \neq 0 \\ \left(1, \operatorname{sgn} \bar{x}_{2}\right) & \text { if } x_{1}=0 \\ \left(\operatorname{sgn} \bar{x}_{1}, 1\right) & \text { if } x_{2}=0 .\end{cases}
$$

Then $(X, \eta)$ is a semi-inner-product space for which the numerical radius norm

$$
|T|_{1}=\sup \{|\langle T x, \eta(x)\rangle|:\|x\|=1\}
$$

is not a Schwarz norm.

It is clear that $[x, y]=\langle x, \eta(y)\rangle$ defines a semi-inner-product on $X$, and it is easy to check that if $T=\left(\begin{array}{l}a 0 \\ c b\end{array}\right)$ has numerical radius $\leqq 1$, then $|b| \leqq 1,|a|+|c| \leqq 1$, and therefore $\|T\| \leqq 1$. This implies that the numerical radius and operator norms agree for each triangular matrix, and hence, by Example 2, the numerical radius norm is not a Schwarz norm in $\mathscr{L}(X)$.

\section{REFERENCES}

1. C. A. Berger and J. G. Stampfli, Mapping theorems for the numerical range (to appear in Amer. J. Math.)

2. C. Foias, Sur certaines theoremes de J. von Neumann concernant les ensembles spectraux, Acta. Sci. Math. (Szeged) 18 (1957), 15-20. 
3. G. Lumer, Semi-inner-product spaces, Trans. Amer. Math. Soc. 100 (1961) 29-43.

4. B. Sz.-Nagy and C. Foias, On certain classes of power-bounded operators, Acta. Sci. Math. 27 (1966), 17-25.

5. J. Von Neumann, Eine Spektraltheorie für allgemeine Operatoren eines unitären Raumes, Math. Nachrichten 4 (1951), 258-281.

6. J. G. Stampfli, Normality and the numerical range of an operator, Bull. Amer. Math. Soc. 72 (1966), 1021-23.

Received March 17, 1967.

INDIANA UNIVERSITY 


\section{PACIFIC JOURNAL OF MATHEMATICS}

\section{EDITORS}

\section{H. ROYDEN}

Stanford University

Stanford, California

\section{J. P. Jans}

University of Washington

Seattle, Washington 98105

\section{J. DUGUNDJI}

Department of Mathematics

Rice University

Houston, Texas 77001

RICHARD ARENS

University of California

Los Angeles, California 90024

\section{ASSOCIATE EDITORS}
E. F. BeCKenbaCH
B. H. NeumanN
F. WOLF
K. YosIDA

\section{SUPPORTING INSTITUTIONS}

UNIVERSITY OF BRITISH COLUMBIA CALIFORNIA INSTITUTE OF TECHNOLOGY UNIVERSITY OF CALIFORNIA MONTANA STATE UNIVERSITY UNIVERSITY OF NEVADA NEW MEXICO STATE UNIVERSITY OREGON STATE UNIVERSITY UNIVERSITY OF OREGON OSAKA UNIVERSITY UNIVERSITY OF SOUTHERN CALIFORNIA
STANFORD UNIVERSITY UNIVERSITY OF TOKYO UNIVERSITY OF UTAH WASHINGTON STATE UNIVERSITY UNIVERSITY OF WASHINGTON

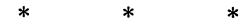

AMERICAN MATHEMATICAL SOCIETY CHEVRON RESEARCH CORPORATION TRW SYSTEMS

NAVAL ORDNANCE TEST STATION 


\section{Pacific Journal of Mathematics}

\section{Vol. 24, No. $1 \quad$ May, 1968}

Harry P. Allen, Lie algebras of type $D_{4}$ over algebraic number fields ...... 1

Charles Ballantine, Products of positive definite matrices. II............ 7

David W. Boyd, The spectral radius of averaging operators ............ 19

William Howard Caldwell, Hypercyclic rings ................... 29

Francis William Carroll, Some properties of sequences, with an application

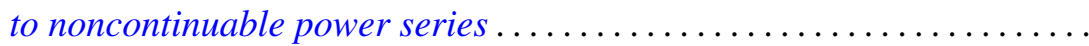

David Fleming Dawson, Matrix summability over certain classes of

sequences ordered with respect to rate of convergence ........... 51

D. W. Dubois, Second note on David Harrison's theory of preprimes. . . . . 57

Edgar Earle Enochs, A note on quasi-Frobenius rings.............. 69

Ronald J. Ensey, Isomorphism invariants for Abelian groups modulo bounded groups ................................ 71

Ronald Owen Fulp, Generalized semigroup kernels ................ 93

Bernard Robert Kripke and Richard Bruce Holmes, Interposition and approximation ................................. 103

Jack W. Macki and James Sai-Wing Wong, Oscillation of solutions to second-order nonlinear differential equations ..................

Lothrop Mittenthal, Operator valued analytic functions and generalizations

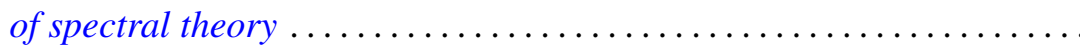

T. S. Motzkin and J. L. Walsh, A persistent local maximum of the pth power deviation on an interval, $p<1 \ldots \ldots \ldots \ldots \ldots \ldots \ldots \ldots \ldots \ldots . \ldots \ldots$

Jerome L. Paul, Sequences of homeomorphisms which converge to homeomorphisms ...........................

Maxwell Alexander Rosenlicht, Liouville's theorem on functions with elementary integrals.

Joseph Goeffrey Rosenstein, Initial segments of degrees .

$\mathrm{H}$. Subramanian, Ideal neighbourhoods in a ring ............

Dalton Tarwater, Galois cohomology of abelian groups . .

James Patrick Williams, Schwarz norms for operators ... .

Raymond Y. T. Wong, A wild Cantor set in the Hilbert cube. 